\title{
Superradiant Instability and Backreaction of Massive Vector Fields around Kerr Black Holes
}

\author{
William E. East ${ }^{1}$ and Frans Pretorius ${ }^{2}$ \\ ${ }^{1}$ Perimeter Institute for Theoretical Physics, Waterloo, Ontario N2L 2Y5, Canada \\ ${ }^{2}$ Department of Physics, Princeton University, Princeton, New Jersey 08544, USA \\ (Received 19 April 2017; revised manuscript received 16 May 2017; published 24 July 2017)
}

\begin{abstract}
We study the growth and saturation of the superradiant instability of a complex, massive vector (Proca) field as it extracts energy and angular momentum from a spinning black hole, using numerical solutions of the full Einstein-Proca equations. We concentrate on a rapidly spinning black hole $(a=0.99)$ and the dominant $m=1$ azimuthal mode of the Proca field, with real and imaginary components of the field chosen to yield an axisymmetric stress-energy tensor and, hence, spacetime. We find that in excess of $9 \%$ of the black hole's mass can be transferred into the field. In all cases studied, the superradiant instability smoothly saturates when the black hole's horizon frequency decreases to match the frequency of the Proca cloud that spontaneously forms around the black hole.
\end{abstract}

DOI: 10.1103/PhysRevLett.119.041101

Introduction.-A remarkable feature of spinning black holes (BHs) is that a portion of their mass - up to $29 \%$ for extremal spin — can, in principle, be extracted. One way to realize this liberation of rotational energy is through the interaction of the $\mathrm{BH}$ with an impinging wave-be it scalar, electromagnetic, or gravitational-with frequency $\omega<m \Omega_{\mathrm{BH}}$, where $\Omega_{\mathrm{BH}}$ is the $\mathrm{BH}$ horizon frequency and $m$ is the azimuthal number of the wave. Waves satisfying this criterion exhibit superradiance and carry away energy and angular momentum from the BH. An analogous phenomenon can occur for charged $\mathrm{BHs}$, where the electromagnetic energy of the $\mathrm{BH}$ is superradiantly transferred to an interacting charged matter field interacting with the $\mathrm{BH}$.

Going back to Ref. [1], there has been speculation of how superradiance could be combined with a confining mechanism to force the wave to continuously interact with the $\mathrm{BH}$ and hence undergo exponential growth-a socalled "black hole bomb." The first nonlinear studies of this process were recently undertaken for a charged scalar field around a charged $\mathrm{BH}$ in spherical symmetry, both in a reflective cavity in asymptotically flat space [2], and in the naturally confining environment of an asymptotically anti-de Sitter domain [3].

However, there is an exciting possibility that a variation of this scenario could, in fact, be realized around astrophysical spinning BHs. Massive bosonic fields with a Compton wavelength comparable to, or larger than, the horizon radius of a $\mathrm{BH}$ can form bound states around the $\mathrm{BH}$, and if the latter is spinning, the bound states can grow from a seed perturbation through superradiance [4-6]. This implies that stellar mass BHs can probe the existence of ultralight bosons with masses $\lesssim 10^{-10} \mathrm{eV}$ that are weakly coupled to ordinary matter and thus difficult to detect by other means. Theoretical scenarios where this might occur include the string axiverse [7,8], the QCD axion [9], and dark photons [10,11]. Such particles could form large clouds, spinning down the $\mathrm{BH}$ in the process. This is of particular interest now that LIGO has begun observing gravitational waves (GWs) [12], since measurements of BH masses and spins from binary mergers can be used to rule out or provide evidence for such particles, in addition to direct searches for the GW signatures of boson clouds $[13,14]$. See [15] for a review.

Though details of the nonlinear growth and saturation of the rotational superradiant instability will be important to help observe or rule out such massive fields, there are presently few results of relevance to this regime where the backreaction on the BH is significant. In Ref. [16], it was found that, for sufficiently large GWs superradiantly scattering off a Kerr $\mathrm{BH}$, backreaction effects decrease the efficiency of energy extraction (for the analogous case of the scattering of a charged scalar field by a ReissnerNordström BH, see [17]). The nonlinear behavior of the superradiant instability of massive bosons has not been addressed before. This is because of the computational cost of solving the equations, in part due to the disparate time scales between the oscillation of the field and the growth rate of the instability and the lack of symmetries to reduce it to a $(1+1)$-dimensional problem (unlike the charged case). Important questions include what the efficiency of energy and angular momentum extraction is, how explosive the nonlinear phase of growth is (e.g., can the energy extraction overshoot limits implied by the parameters of the field and $\mathrm{BH}[2]$ ), and what the final state is after a non-negligible amount of energy has been transferred to the Proca field (e.g., does a stable cloud form around the $\mathrm{BH}$, or could there be something akin to a bosenova where the entire field is rapidly expelled from the vicinity of the $\mathrm{BH}$ ).

In this Letter, we begin to address these questions related to the nonlinear behavior of the superradiant instability of 
massive bosonic fields around a spinning $\mathrm{BH}$. We focus on the case of a vector field, as it exhibits faster growth than a scalar field. The linear regime of the instability for Proca fields has been studied before in various limits $[11,14,18-20]$. Here we find numerical solutions of the full Einstein-Proca field equations. To make the problem computationally tractable, we use a complex field with prescribed $m=1$ azimuthal dependence to give an axisymmetric stress-energy tensor and, hence, spacetime geometry. Beginning with a seed field about a rapidly rotating $\mathrm{BH}$, we find that the instability efficiently grows into the nonlinear regime and smoothly saturates when the $\mathrm{BH}$ horizon frequency decreases to match that of the Proca cloud. This frequency depends on the mass parameter of the field, and for a value near where we expect maximal energy extraction, we find that when the instability saturates a large Proca cloud has formed, containing 9\% of the initial BH mass (and 38\% its initial angular momentum). We use units with $G=c=1$ throughout.

Methodology.-We consider a Kerr BH with initial mass $M_{0}$ and dimensionless spin $a=0.99$ in the presence of a complex Proca field $X^{a}$ with constant mass parameter $\mu$ and numerically evolve the coupled Einstein-Proca equations. The Proca field equation of motion is $\nabla_{a} F^{a b}=\mu^{2} X^{b}$, where $F_{a b}=\nabla_{a} X_{b}-\nabla_{b} X_{a}$, and its corresponding stressenergy tensor is

$$
\begin{aligned}
T_{a b}= & \frac{1}{2}\left(F_{a c} \bar{F}_{b d}+\bar{F}_{a c} F_{b d}\right) g^{c d}-\frac{1}{4} g_{a b} F_{c d} \bar{F}^{c d} \\
& +\frac{\mu^{2}}{2}\left(X_{a} \bar{X}_{b}+\bar{X}_{a} X_{b}-g_{a b} X_{c} \bar{X}^{c}\right)
\end{aligned}
$$

where the overbar indicates complex conjugation. We evolve the Proca equations in a form similar to Ref. [21] (which also evolved the Einstein-Proca equations, though without symmetry restrictions and focusing on nonlinear interactions between the field and a nonspinning $\mathrm{BH}$ ). We restrict to cases where the Proca field has an $m=1$ azimuthal dependence and the resulting stress-energy tensor and spacetime are axisymmetric-i.e., in terms of the Lie derivative with respect to the axisymmetric Killing vector $(\partial / \partial \phi)^{b}, \mathcal{L}_{\phi} X_{a}=i X_{a}$. This allows us to use a twodimensional numerical domain for the spatial discretization, which is essential in making evolutions on time scales of $\sim 10^{5} M_{0}$ computationally feasible. Here we study cases with $\tilde{\mu}:=M_{0} \mu=0.25,0.3,0.4$, and 0.5 . As we discuss below, $\tilde{\mu}=0.25$ is near the value that maximizes the energy extracted from the $\mathrm{BH}$, while $\tilde{\mu}=0.5$ is close to the value that gives the maximum growth rate for the linear instability [22]. We begin with a seed Proca field with energy $\sim 10^{-3} M_{0}$ around a Kerr BH (ignoring the effect of this small field on the initial spacetime geometry) and study the subsequent evolution. We have verified that using different or lower amplitude perturbations gives similar results.
We evolve the Einstein equations using the generalized harmonic formulation, with the gauge degrees of freedom set by fixing the source functions to the values of the initial BH solution in Kerr-Schild coordinates, as in Ref. [16]. To mitigate the accumulation of truncation error during the long period it takes for the Proca field to grow large enough to significantly backreact on the spacetime, we use the background error subtraction technique described in Ref. [23], with the initial isolated spinning $\mathrm{BH}$ as the background solution. As the spacetime evolves, we keep track of the $\mathrm{BH}$ apparent horizon and measure its area $A$ and angular momentum $J_{\mathrm{BH}}$, from which we can derive a mass using the Christodoulou formula

$$
M_{\mathrm{BH}}=\left(M_{\mathrm{irr}}^{2}+\frac{J_{\mathrm{BH}}^{2}}{4 M_{\mathrm{irr}}^{2}}\right)^{1 / 2},
$$

where $M_{\text {irr }}=\sqrt{A / 16 \pi}$ is the irreducible mass. We also measure the flux of Proca field energy $\dot{E}^{H}$ and angular momentum $\dot{J}^{H}$ through the $\mathrm{BH}$ horizon. In addition, for the Proca field we keep track of the energy density $\rho_{E}=-\alpha T_{t}^{t}$ and angular momentum density $\rho_{J}=-\alpha T_{\phi}^{t}$ (where $\alpha=$ $\left[-g^{t t}\right]^{-1 / 2}$ is the lapse), the volume integrals of which give a measure of the total energy $E$ and angular momentum $J$ outside of the BH horizon. Details on the numerical resolution and convergence are given in Supplemental Material [24]; more information on how we evolve the Proca equations, as well as results on the instability in the test-field regime, are provided in Ref. [22].

Results.-All the cases studied here are susceptible to a linear superradiant instability, and after a brief transient period the energy and angular momentum in the Proca field enter a period of exponential growth as shown in Fig. 1. As also shown there, the corresponding loss of mass and angular momentum by the $\mathrm{BH}$, as measured from its horizon properties, closely tracks this. The cases with larger $\mu$ have larger growth rates for the instability and also saturate with smaller energy and angular momentum. Though the mass of the $\mathrm{BH}$ is decreasing in each case, as required by $\mathrm{BH}$ thermodynamics the irreducible mass $M_{\text {irr }}$ is always increasing, and smaller $\mu$ cases saturate with a larger overall increase in $M_{\text {irr }}$.

The reason for the saturation of the superradiant instability is illustrated in Fig. 2, where we plot both the horizon frequency of the $\mathrm{BH} \Omega_{\mathrm{BH}}$ and the ratio of Proca field energy to angular momentum flux through the horizon $\dot{E}^{H} / \dot{J}^{H}$. When $\Omega_{\mathrm{BH}}>\dot{E}^{H} / \dot{J}^{H}$, the superradiant condition is met and the Proca cloud will extract rotational energy from the BH. However, as shown in Fig. 2, eventually the BH's horizon frequency decreases to the point where $\Omega_{\mathrm{BH}} \approx \dot{E}^{H} / \dot{J}^{H}$, and the instability saturates.

We can obtain simple estimates of the final state properties of the black hole if we assume, as roughly consistent with the simulations, that the instability will extract energy 

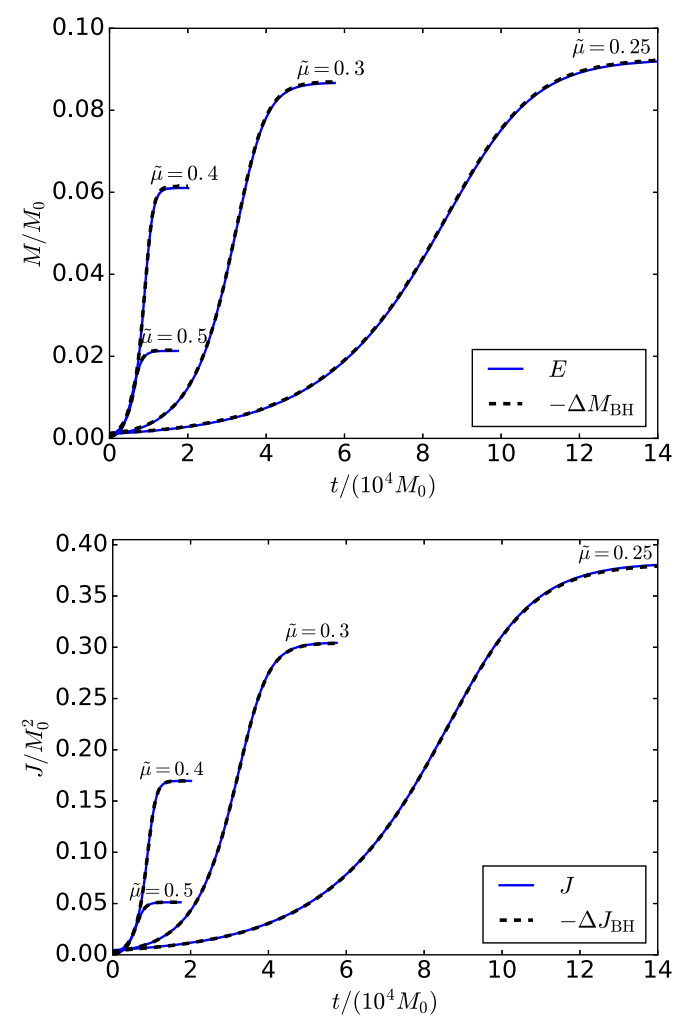

FIG. 1. The energy (top) and angular momentum (bottom) in the Proca field as a function of time (solid lines), along with the loss in mass (top) and angular momentum (bottom) of the $\mathrm{BH}$ (dashed lines).

and angular momentum in some fixed proportion $\omega(\mu)=$ $\dot{E}^{H} / \dot{J}^{H}$ [where $\omega(\mu) \approx \mu\left(1-\tilde{\mu}^{2} / 2\right)$ in the linear and small $\tilde{\mu}$ limit $[25,26]]$ until $\omega(\mu)=\Omega_{\mathrm{BH}}$. We plot the results in Fig. 3, along with the four end-state points from the full nonlinear simulations, showing excellent agreement with the approximation. This indicates an efficient extraction of energy and angular momentum, with a negligible additional increase in irreducible mass (equivalently, $\mathrm{BH}$ entropy).

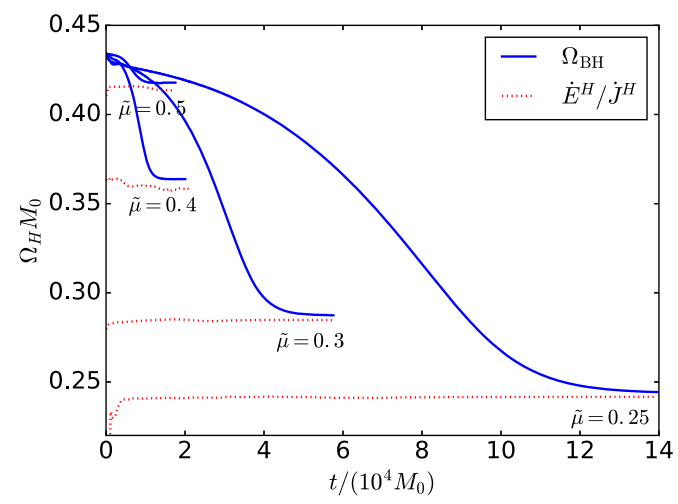

FIG. 2. The BH horizon frequency $\Omega_{\mathrm{BH}}$, as calculated from the BH's mass and angular momentum, and the ratio of the flux of Proca field energy and angular momentum $\dot{E}^{H} / \dot{J}^{H}$ through the $\mathrm{BH}$ horizon, as a function of time.
This is likely due to the relatively slow evolution of the instability compared to the light-crossing time of the $\mathrm{BH}$, even approaching saturation (similar conclusions were reached using a "quasiadiabatic" approximation for the massive scalar field instability in Refs. [27,28]). We see that the energy lost by the $\mathrm{BH}$ should be maximized at $-\Delta M_{\mathrm{BH}} / M_{0} \approx 0.093$, near the value $-\Delta M_{\mathrm{BH}} / M_{0} \approx 0.092$ found for $\tilde{\mu}=0.25$ here. For lower values of $\mu$, less energy but more angular momentum will be extracted, with the instability just converting the Kerr BH into a nonspinning $\mathrm{BH}$ of the same mass in the $\mu \rightarrow 0$ limit.

After saturation, the resulting configuration consists of a BH surrounded by a Proca cloud with roughly stationary energy density, though the phase of the complex field is oscillating at a constant frequency. The energy and angular momentum density of the resulting clouds are illustrated in Fig. 4 for two cases. Away from the BH, the Proca clouds have a roughly spherical energy density, falling off exponentially with distance from the $\mathrm{BH}$. For the larger $\mu$ cases, the cloud is concentrated on much smaller scales near the $\mathrm{BH}$.

As expected, given the close match between the energy and angular momentum lost by the $\mathrm{BH}$ and that gained by the Proca cloud, the radiation from both GWs and the Proca field is negligible (the dominant contribution of which comes from the other modes in the seed perturbation leading to initial radiation). The fact that we are considering a complex Proca field and restricting our study to axisymmetric spacetimes will suppress the gravitational radiation. We can estimate what the gravitational radiation would be for a single real Proca field by using the GW luminosity results from the test field limit [22] and scaling them using $P_{\mathrm{GW}} \propto E^{2}$. This gives $P_{\mathrm{GW}} \sim 6 \times 10^{-8}, 2 \times 10^{-7}$, $6 \times 10^{-7}$, and $6 \times 10^{-8}$ for the Proca field clouds at the end of the $\tilde{\mu}=0.25,0.3,0.4$, and 0.5 simulations, respectively.

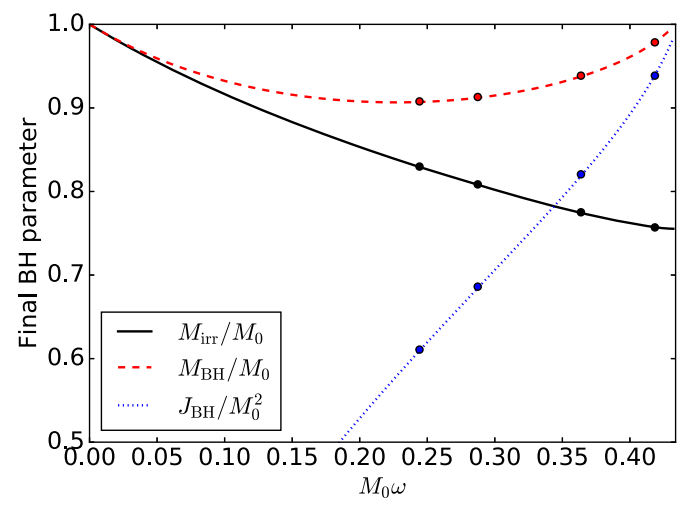

FIG. 3. The final BH irreducible mass, total mass, and angular momentum after saturation of the $m=1$ superradiant instability for a BH with $a=0.99\left(M_{\text {irr, } 0} / M_{0} \approx 0.76\right)$ initially. The lines show the prediction obtained with the assumption that the $\mathrm{BH}$ will lose energy and angular momentum in fixed proportion $\omega$, until $\Omega_{\mathrm{BH}}=\omega$, while the points show the measured values from the simulations. 

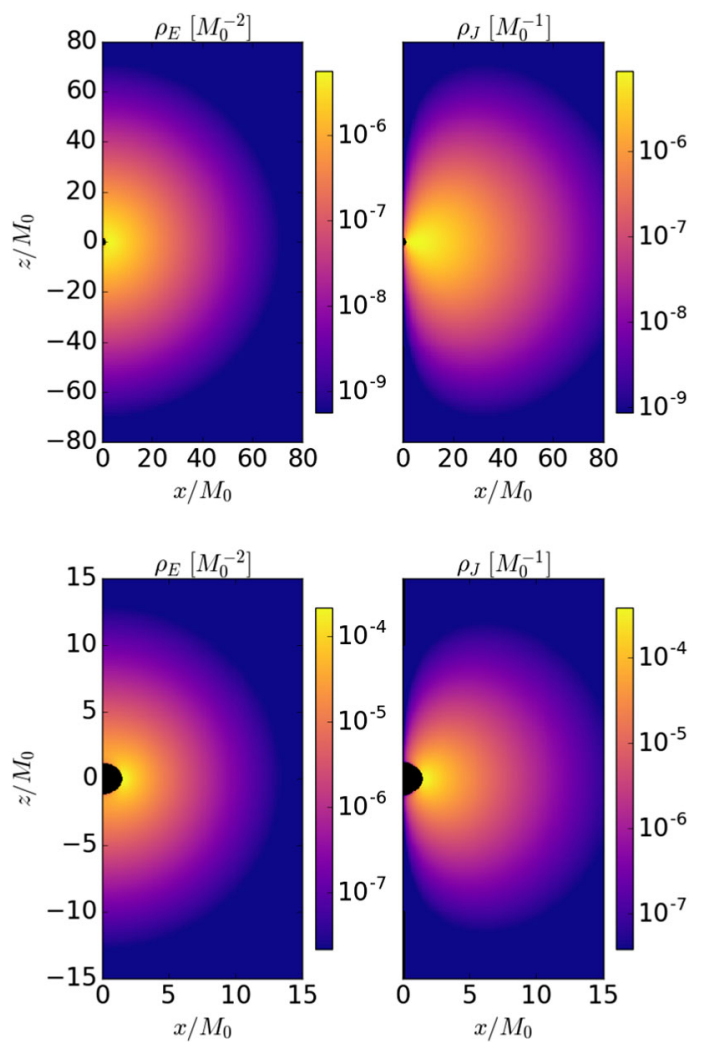

FIG. 4. The energy (left) and angular momentum density (right) of the Proca field in the final state with $\tilde{\mu}=0.25$ (top) and $\tilde{\mu}=$ 0.5 (bottom) for a slice containing the $\mathrm{BH}$ spin axis (the $z$ axis) and perpendicular to the equatorial plane $(z=0)$; note the different scales for the two cases.

This means that, once the $\mathrm{BH}$ has spun down to below the superradiant regime, the Proca cloud will decay via GW emission on time scales of $\sim 10^{5}-10^{6} M_{0}$.

Our Proca field ansatz allows only exploration of the $m=1$ mode instability. Higher $m$ modes are also unstable, even after the $\mathrm{BH}$ has spun down to the point where the $m=1$ becomes stable (ignoring the Proca cloud). However, the grow rates become significantly longer with increasing $m$. For example, for $\tilde{\mu}=0.5$ the $m=2$ instability has a growth rate $\sim 2000$ times as long as the $m=1$ mode [22], and the disparity is worse for smaller $\mu$ (with the relative growth rates scaling as $\mu^{4}$ ).

Conclusion.-We have studied the growth and saturation of the superradiant instability of a massive vector field around a Kerr BH. We find that in all cases the instability efficiently extracts energy from the $\mathrm{BH}$ and then smoothly shuts off as the $\mathrm{BH}$ horizon frequency decreases to the threshold of instability. This contrasts with Ref. [2], where the energy extraction of a charged $\mathrm{BH}$ in a reflecting cavity was seen to overshoot in some cases; this could be due to the presence of multiple unstable modes [3]. We further find that at saturation essentially all the energy and angular momentum extracted from the $\mathrm{BH}$ has gone into forming a cloud of complex Proca "hair" with stationary energy density surrounding the BH. A family of stationary hairy $\mathrm{BH}$ solutions with this property and the same matter model was constructed in Ref. [29] which is plausibly the same as our end states; it would be interesting to investigate in detail how close these solutions are to what we find at saturation. In our case, the Proca clouds persist for the relatively short times we have extended the runs beyond saturation, though this is not adequate to comment on their long-term stability.

We thank Asimina Arvanitaki, Masha Baryakhtar, Stephen Green, Robert Lasenby, Luis Lehner, Vasilis Paschalidis, Justin Ripley, Kent Yagi, and Huan Yang for stimulating discussions. This research was supported in part by Perimeter Institute for Theoretical Physics (W.E.E), the National Science Foundation through Grant No. PHY-1607449 (F. P.), and the Simons Foundation (F. P.). Research at Perimeter Institute is supported by the Government of Canada through the Department of Innovation, Science and Economic Development Canada and by the Province of Ontario through the Ministry of Research, Innovation and Science. Simulations were run on the Perseus Cluster at Princeton University and the Sherlock Cluster at Stanford University.

[1] W. H. Press and S. A. Teukolsky, Nature (London) 238, 211 (1972).

[2] N. Sanchis-Gual, J. C. Degollado, P. J. Montero, J. A. Font, and C. Herdeiro, Phys. Rev. Lett. 116, 141101 (2016).

[3] P. Bosch, S. R. Green, and L. Lehner, Phys. Rev. Lett. 116, 141102 (2016).

[4] T. Damour, N. Deruelle, and R. Ruffini, Lett. Nuovo Cimento 15, 257 (1976).

[5] S. L. Detweiler, Phys. Rev. D 22, 2323 (1980).

[6] T. Zouros and D. Eardley, Ann. Phys. (N.Y.) 118, 139 (1979).

[7] A. Arvanitaki, S. Dimopoulos, S. Dubovsky, N. Kaloper, and J. March-Russell, Phys. Rev. D 81, 123530 (2010).

[8] A. Arvanitaki and S. Dubovsky, Phys. Rev. D 83, 044026 (2011).

[9] A. Arvanitaki, M. Baryakhtar, and X. Huang, Phys. Rev. D 91, 084011 (2015).

[10] B. Holdom, Phys. Lett. B 166, 196 (1986).

[11] P. Pani, V. Cardoso, L. Gualtieri, E. Berti, and A. Ishibashi, Phys. Rev. D 86, 104017 (2012).

[12] B. P. Abbott et al. (Virgo, LIGO Scientific Collaboration), Phys. Rev. Lett. 116, 061102 (2016).

[13] A. Arvanitaki, M. Baryakhtar, S. Dimopoulos, S. Dubovsky, and R. Lasenby, Phys. Rev. D 95, 043001 (2017).

[14] M. Baryakhtar, R. Lasenby, and M. Teo, arXiv:1704.05081.

[15] R. Brito, V. Cardoso, and P. Pani, Lect. Notes Phys. 906, 1 (2015).

[16] W. E. East, F. M. Ramazanoğlu, and F. Pretorius, Phys. Rev. D 89, 061503 (2014).

[17] O. Baake and O. Rinne, Phys. Rev. D 94, 124016 (2016). 
[18] P. Pani, V. Cardoso, L. Gualtieri, E. Berti, and A. Ishibashi, Phys. Rev. Lett. 109, 131102 (2012).

[19] S. Endlich and R. Penco, J. High Energy Phys. 05 (2017) 052.

[20] H. Witek, V. Cardoso, A. Ishibashi, and U. Sperhake, Phys. Rev. D 87, 043513 (2013).

[21] M. Zilhão, H. Witek, and V. Cardoso, Classical Quantum Gravity 32, 234003 (2015).

[22] W. E. East, arXiv:1705.01544.

[23] W. E. East and F. Pretorius, Phys. Rev. D 87, 101502 (2013).
[24] See Supplemental Material at http://link.aps.org/ supplemental/10.1103/PhysRevLett.119.041101 for details on the numerical resolution and convergence.

[25] S. R. Dolan, Phys. Rev. D 76, 084001 (2007).

[26] J. G. Rosa and S. R. Dolan, Phys. Rev. D 85, 044043 (2012).

[27] S. R. Dolan, Phys. Rev. D 87, 124026 (2013).

[28] R. Brito, V. Cardoso, and P. Pani, Classical Quantum Gravity 32, 134001 (2015).

[29] C. Herdeiro, E. Radu, and H. Runarsson, Classical Quantum Gravity 33, 154001 (2016). 